\title{
UNA NUEVA ESPECIE DE TILLANDSIA (BROMELIACEAE) DEL CAÑÓN DE ESCAHUASCO, PUEBLA, MÉXICO
}

\author{
Adolfo Espejo-Serna ${ }^{1,2}$, Ana Rosa López-Ferrari ${ }^{1}$, Jacqueline Ceja-Romero ${ }^{1}$ y \\ Aniceto Mendoza-Ruiz ${ }^{1}$ \\ ${ }^{1}$ Universidad Autónoma Metropolitana-Iztapalapa, División de \\ Ciencias Biológicas y de la Salud, Departamento de Biología, \\ Apdo. postal 55-535, 09340 México, D.F., México. \\ 2Autor para la correspondencia: aes@xanum.uam.mx
}

\section{RESUMEN}

Se describe e ilustra Tillandsia escahuascensis, especie rupícola conocida hasta ahora sólo del cañón de Escahuasco, en el municipio de Tetela de Ocampo, Puebla, México. El nuevo taxon comparte algunas similitudes con T. parryi y $T$. suesilliae, pero se distingue de ellas por sus inflorescencias 2 a 3 veces divididas, con las espigas muy angostamente oblongas a lineares y largamente estipitadas.

Palabras clave: Bromeliaceae, Puebla, taxonomía, Tillandsia.

\begin{abstract}
Tillandsia escahuascensis, a saxicolous species from the Cañón de Escahuasco, municipio de Tetela de Ocampo, Puebla, Mexico, is described and illustrated. The new taxon shares some similarities with T. parryi and T. suesilliae, but it is easily distinguished by its 2-3 branched inflorescences with very narrowly oblong to linear and long stipitate spikes.

Key words: Bromeliaceae, Puebla, taxonomy, Tillandsia.

Como parte del proyecto Bromeliaceae de México hemos venido realizando recolecciones de material botánico de la familia en diferentes regiones del país. Al revisar algunos de los ejemplares provenientes del estado de Puebla nos percatamos de que correspondían a una especie no descrita del género Tillandsia, por lo que proponemos:
\end{abstract}


Tillandsia escahuascensis Espejo, López-Ferrari, Ceja \& A. Mendoza, sp. nov. Fig. 1(A-H).

Herba lithophytica, acaulis, rosulata, florens usque ad $1.5 \mathrm{~m}$ alta; folia numerosa, vaginae distinctae, oblongae, $7-20 \mathrm{~cm}$ longae, $10-11 \mathrm{~cm}$ latae; laminae anguste triangulares, $68-75 \mathrm{~cm}$ longae, $6.5-7.5 \mathrm{~cm}$ latae; pedunculus erectus, teres, $40-60 \mathrm{~cm}$ longus, inflorescentia 2-3 ramifera, spicae peranguste oblongae usque ad lineares, 12-30 cm longae, 1.4-1.5 cm latae, bracteae florales roseae, imbricatae, oblongoellipticae, 4.3-4.5 cm longae, 1.5-1.7 cm latae, ecarinatae, praeter apicem albo-lepidotae; flores distichi, petala violacea, spathulata, 4.8-5.6 cm longa, 9-11 mm lata; stamina et stylus exserta.

Planta saxícola, acaule, de 1.4 a $1.5 \mathrm{~m}$ de alto al florecer, rosetas de tipo tanque, de 60 a $80 \mathrm{~cm}$ de diámetro, solitarias. Hojas numerosas; vainas oblongas, de 7 a $20 \mathrm{~cm}$ de largo, de $10 \mathrm{a} 11 \mathrm{~cm}$ de ancho, pardas castáneas en el haz, densamente punctuladolepidotas en ambas superficies; láminas angostamente triangulares, de 68 a $75 \mathrm{~cm}$ de largo, de 6.5 a $7.5 \mathrm{~cm}$ de ancho en la base, verdes grisáceas, coriáceas, largamente atenuadas en el ápice, densamente blanco-lepidotas en ambas superficies. Inflorescencia terminal, erecta, dos a tres veces ramificada, con 11 a 12 ramas de primer orden; pedúnculo cilíndrico, de 40 a $60 \mathrm{~cm}$ de largo, de 1.4 a $2 \mathrm{~cm}$ de diámetro, de color rosado, cubierto totalmente por las brácteas; brácteas del pedúnculo foliáceas, de 64 a $75 \mathrm{~cm}$ de largo, de 3 a $4 \mathrm{~cm}$ de ancho, verdes grisáceas, blanco-lepidotas en ambas superficies; brácteas primarias foliáceas, angostamente triangulares, reduciéndose gradualmente hacia la porción apical de la inflorescencia, de 10 a $65 \mathrm{~cm}$ de largo, de 1.6 a $3.5 \mathrm{~cm}$ de ancho, verdes, ligeramente rosadas en la base, densamente blanco-lepidotas en ambas superficies; ramas de primer orden de 25 a $45 \mathrm{~cm}$ de largo, con una o dos ramas basales, largamente estipitadas, estipes aplanados, de 6 a $12 \mathrm{~cm}$ de largo, de 5 a $7 \mathrm{~mm}$ de ancho, rosados, lepidotos; espigas muy angostamente oblongas a lineares, aplanadas, de 12 a $30 \mathrm{~cm}$ de largo, de 1.4 a $1.5 \mathrm{~cm}$ de ancho; brácteas florales oblongoelípticas, de 4.3 a $4.5 \mathrm{~cm}$ de largo, de 1.5 a $1.7 \mathrm{~cm}$ de ancho, rosadas, ecarinadas, acuminadas en el ápice, glabras en la porción basal, esparcidamente blanco-lepidotas en la porción apical, glabrescentes en la madurez; flores 5 a 13 por espiga, sépalos libres, angostamente elípticos, de 3.2 a $3.6 \mathrm{~cm}$ de largo, de 9 a $10 \mathrm{~mm}$ de ancho, agudos en el ápice, ecarinados, verdes con el ápice rosado, glabros; pétalos espatulados, de 4.8 a $5.6 \mathrm{~cm}$ de largo, de 9 a $11 \mathrm{~mm}$ de ancho, redondeados en el ápice, blancos en la mitad basal, violáceos en la apical; estambres desiguales, filamentos de 5.8 a $6.5 \mathrm{~cm}$ de largo, blancos en la base, verdes en el ápice; anteras oblongas, de 3 a 4 mm de largo, negras 
Espejo-Serna, et al.: Nueva especie de Tillandsia (Bromeliaceae) del cañón de Escahuasco, Puebla, México
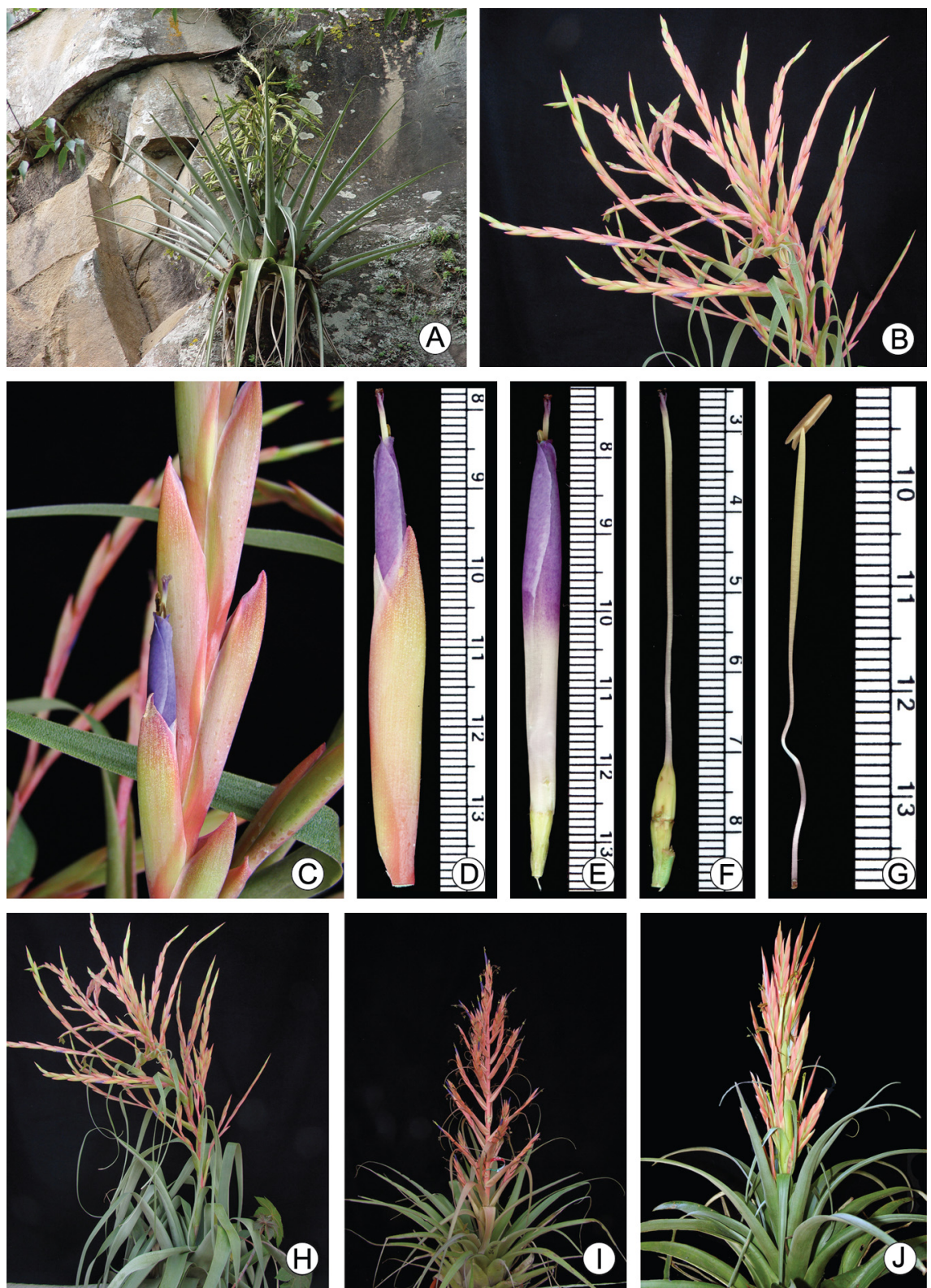

Fig. 1. Tillandsia escahuascensis Espejo, López-Ferrari, Ceja \& A. Mendoza (A-H). A. planta en fructificación en su hábitat; B. inflorescencia en antesis; C. detalle de la flor en la espiga; D. flor completa; E. flor sin los sépalos; F. gineceo; G. estambre, H. planta en antesis (J. Garcia-Cruz et al. 1258); I. Tillandsia parryi Baker (J. Ceja et al. 1961); J. Tillandsia suesilliae Espejo, López-Ferrari \& W. Till (J. Ceja et al. 1745). 
en la madurez; ovario ovoide, de ca. $1 \mathrm{~cm}$ de largo, de 4 a $5 \mathrm{~mm}$ de diámetro, verde, estilo linear, de 5.3 a $5.5 \mathrm{~cm}$ de largo, blanco con el ápice verde, estigma de color violeta, del tipo conduplicado espiral (Brown y Gilmartin, 1989). Cápsula verde a parda clara, fusiforme, rostrada, de 3.7 a $4 \mathrm{~cm}$ de largo, de 5 a $6 \mathrm{~mm}$ de diámetro; semillas pardas oscuras, fusiformes, de 3.7 a $4 \mathrm{~mm}$ de largo, con un apéndice plumoso, blanquecino, de 1.7 a $1.9 \mathrm{~cm}$ de largo.

Tipo: Puebla, municipio de Tetela de Ocampo, en el cañón del río Texocoapa, $3 \mathrm{~km}$ adelante del poblado Cuapancingo, sobre la carretera Chignahuapam - Tetela de Ocampo, 1949'20" N, 9750'28" W, 1723 m, vegetación riparia, 21 junio 2008, florecida en cultivo y herborizada en septiembre de 2010, J. García-Cruz, A. Espejo, A. R. López-Ferrari, J. Ceja y A. Mendoza 1258 (holotipo UAMIZ (10 pliegos)).

Paratipo: Puebla, municipio de Tetela de Ocampo, en el cañón de Escahuasco, cerca de Tetela de Ocampo, 1950'21" N, 97²9'32" W, 1590 m, vegetación riparia, 21 junio 2008, florecida en cultivo y herborizada en septiembre de 2010, J. GarcíaCruz, A. Espejo, A. R. López-Ferrari, J. Ceja y A. Mendoza 1263 (UAMIZ; por distribuir: CICY, IEB, MEXU, XAL).

Tillandsia escahuascensis es conocida hasta ahora sólo del cañón de Escahuasco, en el municipio de Tetela de Ocampo en el estado de Puebla, en donde crece abundantemente sobre los riscos que forman el cañón del río Texocoapa, entre 1590 y $1750 \mathrm{~m}$ s.n.m. La especie empieza a desarrollar las inflorescencias en julio pero la antesis se presenta durante los meses de septiembre y octubre. El epíteto hace referencia al sitio en el cual prospera la especie.

El nuevo taxon presenta algunas similitudes con Tillandsia parryi Baker y $T$. suesilliae Espejo, López-Ferrari \& W. Till (Espejo-Serna et al., 2007) y en material herborizado o fragmentario podría llegar a confundirse, sin embargo se distingue de ellas por sus plantas de gran tamaño, que llegan a medir, en antesis, hasta $1.5 \mathrm{~m}$ de alto y por sus inflorescencias 2 a 3 veces divididas, con las espigas muy angostamente oblongas a lineares y largamente estipitadas. En el Cuadro 1 y la Figura 1 (H-J) es posible apreciar las diferencias entre estas tres especies.

\section{LITERATURA CITADA}

Espejo-Serna, A., A. R. López-Ferrari y W. Till. 2007. Tillandsia suesilliae Espejo, LópezFerrari et W. Till, a new species from central Mexico. Acta Bot. Mex. 78: 85-95. 
Espejo-Serna, et al.: Nueva especie de Tillandsia (Bromeliaceae) del cañón de Escahuasco, Puebla, México

Cuadro 1. Caracteres comparativos de Tillandsia parryi Baker, T. suesilliae Espejo, LópezFerrari \& W. Till y T. escahuascensis Espejo, López-Ferrari, Ceja \& A. Mendoza.

\begin{tabular}{|c|c|c|c|}
\hline & Tillandsia parryi & Tillandsia suesilliae & $\begin{array}{l}\text { Tillandsia } \\
\text { escahuascensis }\end{array}$ \\
\hline Hábito & epífita o saxícola & saxícola & saxícola \\
\hline $\begin{array}{l}\text { Tamaño planta en } \\
\text { antesis }\end{array}$ & $60-80 \mathrm{~cm}$ alto & $60-80 \mathrm{~cm}$ alto & $1.40-1.50 \mathrm{~m}$ alto \\
\hline Vaina foliar & $\begin{array}{l}\text { oblongo-elíptica, } 10 \text { - } \\
18 \mathrm{~cm} \text { largo x } 5.5-10 \\
\mathrm{~cm} \text { ancho, parda } \\
\text { oscura en el haz }\end{array}$ & $\begin{array}{l}\text { oblongo-elíptica, } \\
10.5-13 \mathrm{~cm} \text { largo } \\
\text { x 4-6.5 cm ancho, } \\
\text { verde y teñida de } \\
\text { púrpura en el haz }\end{array}$ & $\begin{array}{l}\text { oblonga, 7-20 } \\
\text { cm largo x 10-11 } \\
\text { cm ancho, parda } \\
\text { castánea en el haz }\end{array}$ \\
\hline Inflorescencia & $\begin{array}{l}\text { una a dos veces } \\
\text { dividida }\end{array}$ & una vez dividida & $\begin{array}{l}\text { dos a tres veces } \\
\text { dividida }\end{array}$ \\
\hline Espigas & $\begin{array}{l}\text { angostamente } \\
\text { elípticas, } 9-24 \mathrm{~cm} \\
\text { largo x } 1.5-2.7 \mathrm{~cm} \\
\text { ancho; estipes } 1.5- \\
1.7 \mathrm{~cm} \text { largo }\end{array}$ & $\begin{array}{l}\text { angostamente } \\
\text { elípticas, } 9.5-18 \mathrm{~cm} \\
\text { largo x } 1.7-2.3 \mathrm{~cm} \\
\text { ancho; estipes } 1.2- \\
1.5 \mathrm{~cm} \text { largo }\end{array}$ & $\begin{array}{l}\text { muy angostamente } \\
\text { oblongas a lineares, } \\
12-30 \mathrm{~cm} \text { largo } \mathrm{x} \\
1.4-1.5 \mathrm{~cm} \text { ancho; } \\
\text { estipes } 6-12 \mathrm{~cm} \\
\text { largo }\end{array}$ \\
\hline Brácteas pedúnculo & $29-40 \mathrm{~cm}$ largo & $14-23 \mathrm{~cm}$ largo & $64-75 \mathrm{~cm}$ largo \\
\hline Brácteas florales & $2.7-3.6 \mathrm{~cm}$ largo & $3.9-5 \mathrm{~cm}$ largo & $4.3-4.5 \mathrm{~cm}$ largo \\
\hline Pétalos & $\begin{array}{l}4.6-5.2 \mathrm{~cm} \text { largo, de } \\
\text { color violeta }\end{array}$ & $\begin{array}{l}5.2-6.1 \mathrm{~cm} \text { largo, } \\
\text { verdes }\end{array}$ & $\begin{array}{l}4.8-5.6 \mathrm{~cm} \text { largo, de } \\
\text { color violeta }\end{array}$ \\
\hline Estigma & violáceo & verde & violáceeo \\
\hline Distribución & $\begin{array}{l}\text { Gto., Hgo., Méx., } \\
\text { N.L., Qro., S.L.P., } \\
\text { Tamps., Tlax. }\end{array}$ & Hgo., S.L.P. & Pue. \\
\hline
\end{tabular}

Brown, G. K. y A. J. Gilmartin. 1989. Stigma types in Bromeliaceae- A systematic survey. Syst. Bot. 14(1): 110-132. 